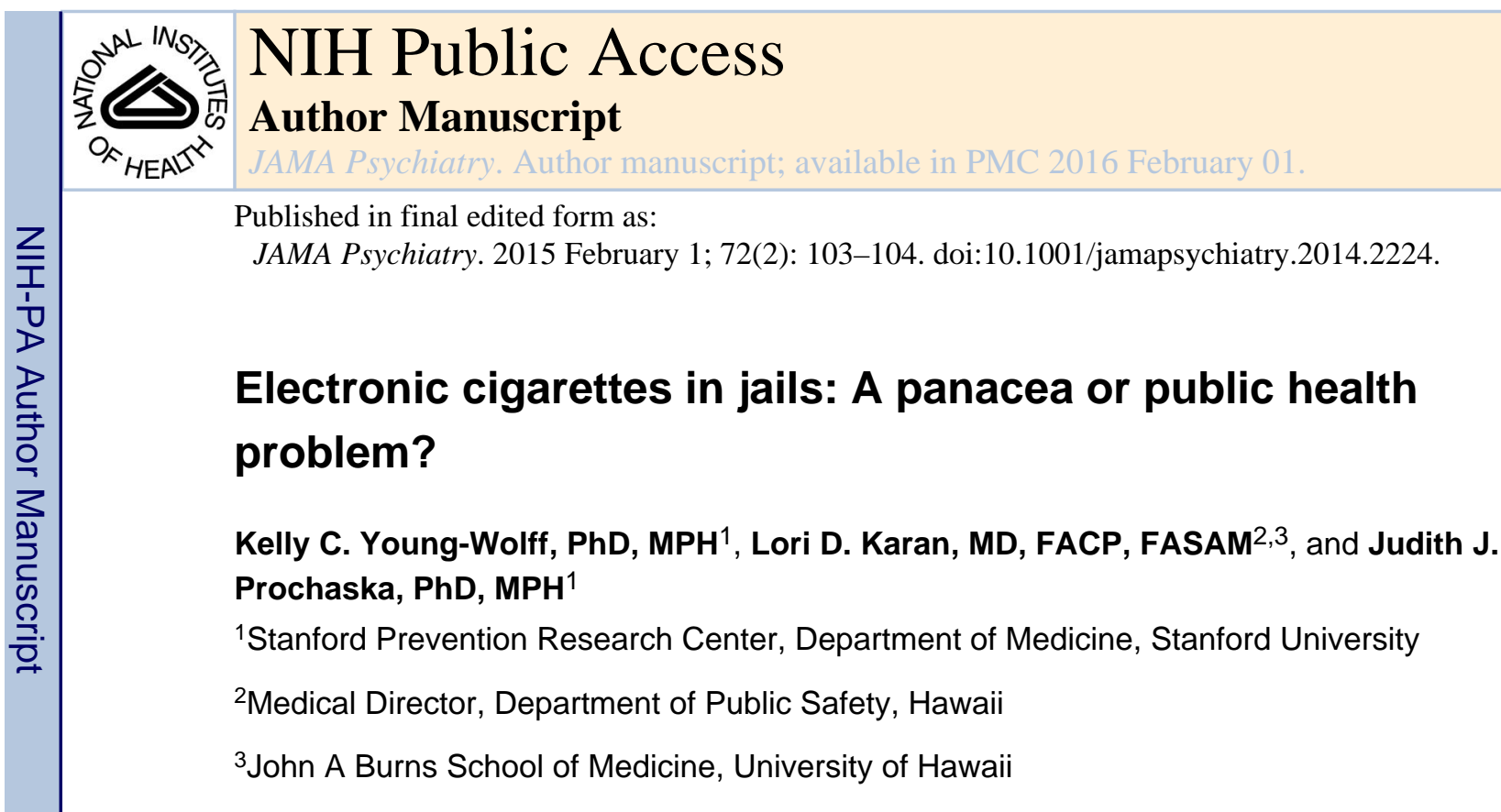

CrossBar electronic cigarettes, sold in "Corrections Orange," regular, and menthol varieties, tout being "The ONLY electronic cigarette designed by a jailer specifically for use in correctional facilities." Transparent packaging is coming soon. Jailcigs ships only to US detention centers declaring exemption from tobacco bans. PrecisionVapor sells electronic cigarettes "designed for institutional use" with plans to produce 'a top quality cigarette that achieves unparalleled success in jail after jail' and prisons in all 50 states with calculated sales of $\$ 9$ per inmate daily or $\$ 328,500$ for 100 inmates annually.

Electronic or e-cigarettes vaporize an "e-juice" composed of water, nicotine, and flavorings in a glycerine solution. Nicotine is delivered to the user when the midst is inhaled. This process avoids burning tobacco with toxic combustion byproducts. The low voltage batteries degrade and are not rechargeable in these correctional products, which are composed of flexible soft plastic casings without metal. E-cigarettes generally enable 500 puffs, equivalent to $40-50$ conventional cigarettes.

Commissaries sell e-cigarettes to inmates according to institutional rules and inmate security levels. Correctional facilities are able to buy e-cigarettes at $\$ 2.50-\$ 3.50$ apiece and sell them for $\$ 8-\$ 30$. Jails in at least eight states are now marketing e-cigarettes. The market is substantial as US jails and prisons currently incarcerate over 2 million people, and a striking 11.6 million cycle through the jail system annually. Generated revenue reported for the jail facilities has been significant. ${ }^{1}$ Given budgetary cutbacks, e-cigarette sales are increasingly used to fund inmate educational and rehabilitation programs and to supplement the salaries of correctional officers. Staff members who previously took smoke breaks can now inhale ecigarettes without leaving their posts, increasing the efficiency of facility operations. Those who advocate for e-cigarettes assert that inmates are calmed, morale is boosted, and that

Correspondence to: Judith J. Prochaska, PhD, MPH, Stanford University, Medical School Office Building, X316, 1265 Welch Road, Stanford, CA 94305-5411, phone: 650-724-3608, Fax 650-725-6247.JPro@ Stanford.edu.

Disclosures: Drs. Young-Wolff and Karan have no conflicts of interest to disclose.

Additional information: The views expressed in this article are those of the authors and do not necessarily represent the views of the Department of Public Safety, Hawaii.

Additional Contributions: We thank Lisa Henriksen, PhD, Trent Johnson, MPH, our research team, and three anonymous reviewers for providing editorial review. 
contraband is less. Custody officers tout using the 'privilege' of e-cigarettes to control and/or punish those who 'misbehave.'

\section{What is wrong with this picture?}

First and foremost, nicotine is a psychoactive and addictive drug that acts along the same brain motivational/reward pathways as other substances. While it is the combustion products of tobacco and additives in cigarettes that lead to cancer, heart, and lung disease, it is nicotine that alters mood and leads to repeated self-administration and relapse. The rapidity of inhaled nicotine delivery through the lungs directly to the brain makes it extremely reinforcing. When combined with the ability to self-adjust dosage by the duration and intensity with which one puffs, individuals can immediately modulate their mood. Smokers rely upon 'hits' of nicotine to alter their feelings, which then becomes repeatedly conditioned, via internal and external cues, leading to vicious cycles of addiction and relapse.

Over $70 \%$ of the US correctional population has an addiction and/or mental illness. Jails and prisons have become a healthcare safety net for this vulnerable population due to the 'War on Drugs,' mandatory drug sentencing, and de-institutionalization of the mentally ill. Individuals with mental illness have a high prevalence of smoking and nicotine dependence, low rates of smoking cessation, and are disproportionately impacted by smoking-related morbidity and mortality. Quitting smoking is associated with long-term reductions in depression, anxiety, and stress and with enhanced positive mood after the effects of nicotine withdrawal dissipate, as demonstrated by a recent meta-analysis, with measured improvements in well-being similar in magnitude to the effectiveness of anti-depressant medications. ${ }^{2}$ Persons who quit cigarettes may fare better in their recovery from all drugs, ${ }^{3}$ including once they are released from incarceration. Introducing e-cigarettes into previously smoke-free jail settings will likely renormalize smoking and cue craving among former smokers in this vulnerable population, prolong nicotine dependence, and possibly increase return to traditional cigarettes after leaving jail. Further, e-cigarette introduction may lead to initiation of these products among never smokers who become incarcerated.

Still unsupported, one of the arguments for e-cigarettes is they may aid tobacco cessation. If studies find e-cigarettes assist smokers in quitting use of traditional cigarettes, they may be acceptable in correctional facilities that continue to sell traditional cigarettes. However, introduction of e-cigarettes in smoke-free correctional facilities, where cues to use have been removed, is counter-productive. In a recent survey of 40 prisoners following a ban on traditional cigarettes, one inmate described, "If I know I can't get it [cigarettes], it's not going to bother me. If I know it's around me, and I can smell it or something, then it bothers me because I know I can get it if I want it ...". A relevant parallel, psychiatric and drug treatment units over the past two decades that have adopted complete smoking bans have been successful, with reports of less aggressive behaviors and stabilization of patients' moods in comparison to the chaos experienced with none or partial bans. ${ }^{5}$

Although e-cigarettes contain fewer harmful constituents than conventional cigarettes, there is large variability in their nicotine content and design and quality of manufacturing. E- 
cigarettes thus far have escaped FDA regulation and the long-term consequences of firstand secondhand exposure to e-cigarettes are largely unknown. It is estimated that $20-27 \%$ of the propylene glycol and vegetable glycerin-based liquid particles are inhaled through the lungs and deposited into the circulatory system. ${ }^{6}$ Heavy metals including tin, nickel, and chromium from the heating elements also stream deep into the alveoli. ${ }^{6}$ While bystanders are not exposed to side-stream smoke, they do take in the exhaled aerosol. Further, nicotine deposits and other residues from the vapor accumulate on indoor surfaces and can be absorbed transdermally. The questionable constituents and health effects of e-cigarette exposures are likely magnified in the confined and crowded quarters of jail settings.

E-cigarettes in jails and prisons pose additional unique concerns. The soft plastic tubing of e-cigarettes can hide contraband and be ingested or inserted anally. E-cigarettes also can be used to covertly smoke other substances, including marijuana, opium, and crack cocaine. When inmates work, they earn as little as 15 -cents per hour, so not all inmates can afford to purchase commissary items. "Selling like hotcakes"1 is a danger signal, and reliance upon ecigarette sales to address budget shortfalls is convoluted. Most inmates enter jail addicted to nicotine, and the potential for exploitation is high. Further, any psychoactive substance, including nicotine, will engender a black market. Of concern, if correctional facilities become financially reliant on the income from e-cigarettes for officers' salaries and programming, it will be increasingly difficult for these facilities to revert to nicotine-free environments even if the public health harms become more apparent.

Jails and prisons are uniquely situated to treat nicotine addiction and connect an extremely hard-to-reach, confined population with cessation and relapse prevention services. The majority of incarcerated adults are low-income, ethnic/racial minorities, with a high prevalence of mental health problems; ${ }^{7}$ the same groups identified as priority populations for tobacco control. Although fewer than $20 \%$ of US correctional facilities have provided FDA approved cessation medications and counseling, ${ }^{7}$ the Affordable Care Act now requires Medicaid coverage of tobacco cessation medications for US-born or naturalized incarcerated individuals. Encouragingly, recent evidence indicates that low-cost, behavioral counseling focused on identifying triggers and developing coping skills reduced relapse to smoking amongst newly released felons. ${ }^{8}$ Preventing and treating tobacco use is a National Commission of Correctional Health standard. With the advent of 'vaping', however, this policy has become outdated, focusing only on "tobacco." From a cost-benefit perspective, treating nicotine addiction offers broad, longer-term benefits with few risks relative to the immediate fiscal gains and potential dangers and misuse of e-cigarettes.

\section{Acknowledgments}

Dr. Prochaska is Principal Investigator on an Investigator Initiated Research Award funded by Pfizer and has provided grant review and ad hoc advisory to the company.

Funding: This research was supported by grants from the National Institute of Mental Health (\#RO1 MH083684), the National Heart, Lung, and Blood Institute (\#T32 HL007034-37 and \#R01 HL117736-01), and the National Institute on Drug Abuse (\#P50 DA09253). The funders played no role in the preparation, review, or approval of the manuscript. 


\section{References}

1. New York Times. Rural Jails, E-Cigarettes Are a Calming Vapor. http://www.nytimes.com/ 2014/01/24/us/in-rural-jails-a-calming-vapor.html?_r=0.

2. Taylor G, McNeill A, Girling A, Farley A, Lindson-Hawley N, Aveyard P. Change in mental health after smoking cessation: systematic review and meta-analysis. BMJ. [published online February 13, 2014). doi: http://dx.doi.org/10.1136/bmj.g1151.

3. Hall SM, Prochaska JJ. Treatment of smokers with co-occurring disorders: emphasis on integration in mental health and addiction treatment settings. Annu Rev Clin Psychol. 2009; 5:409-431. [PubMed: 19327035]

4. Thibodeau L, Seal DW, Jorenby DE, Corcoran K, Sosman JM. Perceptions and Influences of a State Prison Smoking Ban. J Correct Health Care. 2012; 18(4):293-301. [PubMed: 22935623]

5. Lawn S, Pols R. Smoking bans in psychiatric inpatient settings? A review of the research. Aust N Z J Psychiatry. 2005; 39(10):866-885. [PubMed: 16168014]

6. Grana R, Benowitz N, Glantz S. E-Cigarettes: A Scientific Review. Circulation. 2014; 129:19721986. [PubMed: 24821826]

7. Cork K. Tobacco behind bars: Policy options for the adult correctional population. Public Health Law Center. 2012

8. Clarke JG, Stein LA, Martin RA, Martin SA, Parker D, Lopes CE, McGovern AR, Simon R, Roberts M, Friedman P, Bock B. Forced smoking abstinence: not enough for smoking cessation. JAMA Intern Med. 2013; 173(9):789-794. [PubMed: 23567902] 